\title{
Análise Fatorial Confirmatória do Extended Class Play numa Amostra Portuguesa de Jovens Adolescentes
}

\author{
Confirmatory Factor Analysis of the Extended Class Play on a Sample \\ of Portuguese Young Adolescents
}

\author{
João V. Correia ${ }^{*}, a$, António J. Santos ${ }^{a}$, Miguel Freitas ${ }^{a}$, António Rosado $^{b}$ \& Kenneth Rubin ${ }^{c}$ \\ ${ }^{a} I S P A$ - Instituto Universitário de Ciências Psicológicas, Sociais e da Vida, Lisboa, Distrito de Lisboa, Portugal, \\ ${ }^{b}$ Faculdade de Motricidade Humana, Cruz Quebrada, Distrito de Lisboa, Portugal \\ $\&{ }^{c}$ University of Maryland, College Park, Maryland, USA
}

\begin{abstract}
Resumo
O Extended Class Play (ECP; Burgess, Rubin, Wojslawowicz, Rose-Krasnor, \& Booth, 2003) procura avaliar, através dos relatos dos pares, a reputação social dos sujeitos. O objetivo deste trabalho é testar a estrutura hexafatorial recentemente proposta, averiguando igualmente a sua adequação para género e idade, numa amostra portuguesa de 465 adolescentes (230 raparigas). Uma Análise Fatorial Confirmatória revelou que o modelo de seis fatores apresenta bons índices de ajustamento, de fiabilidade e validade e ainda boa adequação para gênero e idade. Estes resultados são consistentes com outros trabalhos, ao sugerir que o modelo hexafatorial do ECP apresenta capacidades psicométricas aceitáveis, constituindo-se como uma medida válida a utilizar na investigação sobre a reputação e o funcionamento sociais de adolescentes.

Palavras-chave: Relações de pares, reputação social, adolescência, Extended Class Play.
\end{abstract}

\begin{abstract}
The Extended Class Play (ECP) assesses individuals' social reputation through peer reports. In this study, the recently presented six-factor structure was tested on a Portuguese sample of 465 adolescents (230 girls). A Confirmatory Factor Analysis demonstrated good fit of the data to this six-factor structure, good composite reliability and validity. Results also showed good fit to both genders and age groups. Therefore, results are consistent with previous research and suggest that this six-factor structure has good psychometric characteristics and that the ECP seems to be a valid measure for investigation on adolescents' social functioning and reputation.

Keywords: Peer relationships, social reputation, adolescence, Extended Class Play.
\end{abstract}

Na adolescência registam-se uma série de mudanças biológicas, cognitivas, emocionais e, também, sociais. De facto, neste período, as interações com os pares tornam-se mais complexas, multifacetadas e diferenciadas (Rubin, Bukowski, \& Parker, 2006), observando-se igualmente um crescimento exponencial do tempo passado em interação social com os pares - numa maior diversidade de atividades e contextos e com menor supervisão de adultos - e da partilha de intimidade com os outros que lhes são significativos (Gifford-Smith \& Brownell, 2003; Sullivan,

\footnotetext{
" Endereço para correspondência: ISPA-IU, Rua Jardim do Tabaco, Lisboa, Portugal 1149-041. E-mail: joao.v.correia@sapo.pt, asantos@ispa.pt,mfreitas@ispa. pt e krubin@umd.edu

Os autores gostariam de agradecer a todas os jovens que aceitaram participar neste estudo, financiado em parte pela F.C.T. (PTDC/PSI-PDE/098257/2008). Os autores gostariam ainda de agradecer a todos os colegas da linha 1, Psicologia do Desenvolvimento, da UIPCDE pelos seus comentários valiosos.
}

1953). Por outro lado, registam-se igualmente alterações ao nível do grupo de pares, concretamente na sua estrutura, tamanho e composição, uma vez que passam a incluir também membros do sexo oposto. Também a pertença a um grupo assume uma enorme importância para os adolescentes (Rubin, Coplan, Chen, Buskirk, \& Wojslawowicz, 2005), concretamente para o seu bem-estar emocional, validação pessoal e desenvolvimento de um autoconceito positivo (Harter, 1993; Sullivan, 1953), bem como para a sua capacidade para lidar com o stress (Hartup, 1992) e, ainda, para o seu bom ajustamento futuro (Bagwell, Newcomb, \& Bukowski, 1998).

Assim, este afigura-se como um período particularmente importante e desafiante (Roeser, Eccles, \& Sameroff, 2000), em que se contam entre as principais tarefas desenvolvimentais a integração e o estabelecimento de relações positivas no seio do grupo de pares, a formação de relações mais próximas e íntimas de amizade ou de cariz romântico (Masten \& Coatsworth, 1998). Nesta fase, tornam-se então especialmente salientes as preocupações 
Correia, J. V., Santos, A. J., Freitas, M., Rosado, A. \& Rubin, K. (2014). Análise Fatorial Confirmatória do Extended Class Play numa Amostra Portuguesa de Jovens Adolescentes.

relativas à aceitação social, ao bullying e à vitimização (Rubin, Bukowski, et al., 2006).

Com efeito, o envolvimento em interações sociais e o seu sucesso são alvos de expectativas e julgamentos, por parte tanto dos adultos como de outras crianças e adolescentes (Masten, 2005). Deste modo, se o fracasso em cumprir tais expectativas pode ser um critério de identificação de um problema ou de dificuldades, como o bullying ou a retirada social (Masten \& Curtis, 2000), pelo contrário, o sucesso em tarefas desenvolvimentais relativas ao funcionamento social com os pares pode ser um critério de competência e resiliência para professores, pais e investigadores - ou até para o próprio jovem (Masten, 2005). Ou seja, estes aspectos poderão ser importantes indicadores de adaptação ou desadaptação individual e, na medida em que o funcionamento num dado domínio tende a ter implicações noutras áreas, a competência em tarefas desenvolvimentais salientes nesta idade poderá permitir a avaliação do desenvolvimento dos adolescentes (Burt, Obradović, Long, \& Masten, 2008).

Centrando-nos sobre a reputação social, isto é, sobre a forma como os indivíduos são percecionados pelos seus pares, a investigação tem procurado avaliar quais os comportamentos, atitudes e características que levam à formação de determinada reputação social e concluído que, uma vez estabelecida, tende não só a regular as relações entre os pares, como também a ser preditiva da futura adaptação psicossocial dos sujeitos (Morison \& Masten, 1991; Prinstein, Rancourt, Guerry, \& Browne, 2009; Rubin, Chen, McDougall, Bowker, \& McKinnon, 1995). Um dos métodos mais utilizados para descrever quer o comportamento social, quer a reputação social dos sujeitos consiste nas nomeações de pares, procedimento que se tem revelado válido em diferentes culturas (e.g. Casiglia, Lo Coco, \& Zappulla, 1998; Chen, Rubin, Li, \& Li, 1999) e particularmente útil na adolescência. De forma geral, pede-se aos sujeitos que indiquem o(s) colega(s) que melhor personifica $(\mathrm{m})$ uma série de papéis ou descrições comportamentais de natureza variada, como manifestações de agressividade, retirada social, vitimização ou comportamento pró-social.

A principal vantagem deste método prende-se com o facto de confiar em vários informadores - os diferentes colegas - e que estes, que partilham uma história de múltiplas interações em diversos contextos com os sujeitos, são os melhores conhecedores dos seus comportamentos sociais e aqueles que determinam, em última análise, a sua integração, estatuto e reputação social (Hymel \& Rubin, 1985). Por outro lado, o facto de a reputação social tender para a estabilidade poderá ser encarado como uma potencial desvantagem deste procedimento, uma vez que as avaliações dos colegas poderão estar, de algum modo, enviesadas. Efetivamente, um sujeito pode ter já cessado ou modificado um determinado comportamento social, mas a sua reputação tende a permanecer e a resistir à mudança (Hymel, Wagner, \& Butler, 1990), algo particularmente evidente nas manifestações de agressividade (e.g., Moskowitz, Schwartzman, \& Ledingham, 1985).

\section{Revised Class Play}

O Revised Class Play (Masten, Morison, \& Pellegrini, 1985) tem sido uma das medidas mais utilizadas para avaliar o funcionamento e reputação social, bem como uma série de questões relacionadas com a competência social, sobretudo a partir da pré-adolescência. Este instrumento é uma extensão do Class Play (Lambert \& Bower, 1961 cit. por Masten et al., 1985) que, na sua versão original, evidenciou estabilidade a curto-prazo, bem como validade concorrente - correlacionando-se com desajustamento emocional e comportamental - e preditiva - antecipando futuras dificuldades académicas e psicopatológicas de forma mais precisa do que os autorrelatos, relatos dos professores ou desempenho académico.

Ainda assim, Masten et al. (1985) procuraram desenvolver um método de avaliação da reputação social que: (a) revelasse melhores propriedades psicométricas; (b) pudesse distinguir diferentes dimensões da reputação social, incluindo também os seus aspetos positivos (que se encontravam pouco representados na versão original); (c) explorasse melhor as relações entre estas dimensões e outras medidas de competência académica; (d) analisasse a relação entre a reputação social e padrões de competência ou desajustamento sociais.

Deste modo, os autores criaram uma nova versão do Class Play - o Revised Class Play (RCP) -, introduzindo algumas modificações na estrutura, no conteúdo e na forma de aplicação. Assim, a nova medida passou a ser composta por 30 papéis - 15 de valência negativa e 15 de valência positiva -, de modo a não só incluir aspetos positivos da competência social, como também a criar uma bipolaridade nos atributos sociais descritos. Para evitar possíveis enviesamentos na avaliação dos professores e dos pares, foram retirados os itens relativos à competência académica ou intelectual, enquanto outros foram simplificados. Finalmente, foi anexada uma lista de todos os membros da turma, procurando facilitar a inclusão de todos os colegas (Masten et al., 1985).

Com base numa amostra composta por 612 sujeitos, alunos entre o $3^{\circ}$ e o $6^{\circ}$ ano de escolaridade, os autores realizaram uma análise fatorial de componentes principais com rotação varimax que revelou uma estrutura de 3 dimensões ortogonais que explicavam $64 \%$ da variância total. Todos os itens positivos saturaram substancialmente no primeiro fator - designado por Sociabilidade-Liderança - , enquanto os itens negativos dividiram-se nos restantes fatores, denominados de Agressivo-Disruptivo e Sensível-Isolado. Esta estrutura fatorial foi confirmada numa segunda amostra independente, e em análises separadas para género e ano escolar, tendo revelado boa consistência interna ( $\alpha$ entre 0,85 e 0,95 ) e estabilidade ao longo do tempo (Morison \& Masten, 1991). Adicionalmente, os 3 fatores apresentaram correlações entre si de muito fracas 
a moderadas - sugerindo tratarem-se de constructos relativamente independentes - $\mathrm{e}$ associações com medidas de adaptação psicossocial na adolescência (Morison \& Masten, 1991).

Contudo, investigações realizadas em diferentes contextos culturais - por exemplo, China (Chen, Rubin, \& Sun, 1992) ou Itália (Casiglia et al., 1998) - revelaram não só uma inadequação da estrutura de três fatores proposta por Masten e colaboradores (1985), como também a existência de modelos distintos para cada realidade considerada, realçando assim a importância e necessidade de se considerar a especificidade cultural no estudo da reputação social. Também Zeller, Vannatta, Schafer e Noll (2003) não obtiveram suporte empírico para esta estrutura original numa análise fatorial confirmatória, o que os levou a defenderem a necessidade de se discriminar melhor os diferentes comportamentos sociais.

\section{Extended Class Play}

Deste modo, a partir do RCP (Masten et al., 1985), Burgess, Rubin, Wojslawowicz, Rose-Krasnor e Booth-LaForce (2003) desenvolveram uma nova versão, mais longa, que pudesse concretizar esta melhor diferenciação entre os vários comportamentos sociais listados. Assim, acrescentaram dez novos itens, com o objetivo de melhor captar diferentes tipos de agressividade e isolamento social, bem como avaliar a vitimização pelos pares. Mais especificamente, foram adicionados dois itens relativos a Agressividade(e.g. "Espalha boatos sobre os outros para que os colegas deixem de gostar deles"), procurando distinguir diferentes tipos: físico, relacional e verbal. Acrescentaram-se igualmente cinco itens relativos ao Isolamento Social (e.g. "Fala pouco, fala baixo"), de modo a poder diferenciar dois fenómenos conceptualmente distintos: o da retirada social do grupo de pares (motivado pela timidez, ansiedade ou desinteresse social) e o do isolamento ativo promovido pelo grupo de pares (devido a comportamentos socialmente desadequados ou pouco aceitáveis, como a agressividade). Por último, foram adicionados três itens que pudessem captar comportamentos de Vitimização (e.g. "É frequentemente ofendido/ insultado").

Com base numa amostra de estudantes dos $5^{\circ}$ e $6^{\circ}$ anos de escolaridade, uma análise exploratória de componentes principais com rotação varimax deduziu a existência de 5 fatores ortogonais (Burgess et al., 2003; Burgess et al., 2004): Agressividade (9 itens; $\alpha=0,91$ ); Timidez/ Retirada social ( 6 itens; $\alpha=0,82)$; Vitimização/ Exclusão ( 8 itens; $\alpha=0,87$ ); Comportamento pró-social (6 itens; $\alpha=0,82$ ); Popularidade/ Sociabilidade (5 itens; $\alpha=0,87$ ). Refira-se que, dos dez itens adicionados por Burgess et al. (2003), sete não foram incluídos na estrutura final de 5 fatores, por apresentarem pesos fatoriais inconsistentes.

O Extended Class Play (ECP) revelou fiabilidade, validade e estabilidade em diferentes momentos de avaliação, com recurso a uma amostra normativa de 827 crianças que frequentavam o $5^{\circ}$ ano de escolaridade (Burgess et al., 2003). Este modelo foi posteriormente suportado por uma análise fatorial confirmatória $-\chi 2=2196,47, g l=0,395$ $(p<0,001), \mathrm{CFI}=0,86, \mathrm{RMSEA}=0,08, \mathrm{e} \mathrm{SRMR}=0,09$ (Burgess et al., 2003; Burgess et al., 2004) - e, desde então, tem-se revelado válido não só em diferentes estudos (e.g. Wojslawowicz, Rubin, Burgess, Booth-LaForce, \& Rose-Krasnor, 2006), como também em diferentes realidades culturais (e.g. Menzer, Oh, McDonald, Rubin, \& Dashiell-Aje, 2010).

Ainda assim, mais recentemente, alguns autores têm procurado separar o fator da Vitimização/ Exclusão em duas dimensões distintas (e.g. Menzer et al., 2010). De facto, e apesar de ambas estarem associadas a desajustamento futuro (e.g. Gazelle \& Rudolph, 2004), o fenómeno da vitimização consiste em ser alvo de agressividade física ou verbal por parte dos pares (Buhs, Ladd, \& Herald-Brown, 2010), enquanto o da exclusão diz respeito a comportamentos de rejeição que passam pelo evitar, ignorar ou mesmo excluir ativamente os sujeitos das atividades do grupo (Gazelle \& Ladd, 2003). Estes dois fenómenos parecem ter não só diferentes causas, como também consequências no ajustamento distintas, na medida em que a vitimização surge relacionada com a agressividade e também com o evitamento da escola, enquanto a exclusão está mais associada à retirada social e a uma diminuição na participação das actividades escolares (Buhs et al., 2010; Menzer et al., 2010). Assim, foi proposto um modelo composto por 6 fatores: Agressividade ( 9 itens; $\alpha=0,91$ ); Timidez/ Retirada social (6 itens; $\alpha=0,81$ ); Comportamento pró-social (6 itens; $\alpha=0,82$ ); Popularidade/ Sociabilidade (5 itens; $\alpha=$ 0,87 ); Vitimização ( 3 itens $\alpha=0,91$ ); e Exclusão ( 3 itens; $\alpha$ $=0,90)$. Nesta nova estrutura, dois itens anteriormente pertencentes ao fator Vitimização/ Exclusão foram excluídos ("É facilmente melindrável ou ofendido"; "Habitualmente está triste") por não caracterizarem particularmente bem qualquer um dos fenómenos (Menzer et al., 2010).

\section{Diferenças Culturais, de Género e de Idade}

Vários estudos têm atestado a variabilidade na avaliação do comportamento social consoante o contexto cultural, o género ou a idade considerados. Relativamente ao contexto cultural, trabalhos de cariz sociológico (e.g. Trentini \& Muzio, 1995) têm procurado caracterizar os valores mais importantes em amostras representativas de diferentes países, tendo concluído que os Portugueses e os Italianos atribuem grande importância às relações sociais, ao altruísmo e a criatividade - e menor destaque a valores como a autoridade, o prestígio e a progressão na carreira, mais frequentes entre os povos Americano e Canadiano. A investigação tem igualmente demonstrado que os comportamentos pró-sociais (e.g. ajudar, partilhar, apoiar, entre outros) na interação com pares e pais são mais prevalentes nas culturas orientais do que nas ocidentais, enquanto a agressividade (física, verbal ou relacional) se encontra mais presente nestas últimas (Chen, Chung, \& Hsiao, 2009). Por outro lado, os trabalhos de Chen e colaboradores (Chen et al., 1999; Chen et al., 1992) concluíram que o comportamento de retirada social, francamente desencorajado e 
Correia, J. V., Santos, A. J., Freitas, M., Rosado, A. \& Rubin, K. (2014). Análise Fatorial Confirmatória do Extended Class Play numa Amostra Portuguesa de Jovens Adolescentes.

associado a risco de futuro desajustamento psicossocial nas culturas ocidentais, é não só aceite, como também valorizado por pais, professores e pares e, ainda, positivamente associado com competência e aceitação social, bem como com sucesso académico na cultura chinesa. Neste sentido, os investigadores têm proposto que as normas culturais ou étnicas poderão contribuir para as diferentes associações entre comportamentos sociais, como, por exemplo, a rejeição com a agressão ou a retirada social (Rubin, Cheah, \& Menzer, 2010).

A literatura tem indicado igualmente a existência de diferenças no funcionamento e na aceitação de determinados comportamentos sociais consoante o género considerado. Efetivamente, os rapazes parecem estar mais preocupados com o seu estatuto social no grupo de pares, enquanto as raparigas procuram a afiliação em pequenos grupos nos quais a intimidade é maior (e.g. Rose \& Smith, 2009; Rubin, Wojslawowicz, Rose-Krasnor, Booth-LaForce, \& Burgess, 2006). De igual modo, a agressividade parece ser mais aceitável nos rapazes (Rose \& Smith, 2009), enquanto a retirada social é mais tolerada para o género feminino, acarretando menos riscos de ajustamento futuro (e.g., Rubin, Coplan, \& Bowker, 2009) - ainda que estes resultados provenham essencialmente de estudos realizados com crianças, existindo muito menos evidência conclusiva quando se foca a adolescência (Gazelle \& Rudolph, 2004) ou outras culturas que não a norte-americana.

Finalmente, poucos estudos procuraram evidência empírica para medidas de reputação social em diferentes grupos de idade ou se focaram especificamente na adolescência, e os que o fizeram não só utilizaram apenas o RCP, como não encontraram suporte empírico para a estrutura fatorial proposta (Luthar \& McMahon, 1996; Zeller et al., 2003). Perante a evolução registrada neste instrumento de avaliação da reputação social através das nomeações dos pares, as especificidades culturais que têm sido evidenciadas, as diferenças de género registradas e a relativa escassez de estudos para diferentes idades - em particular para a adolescência -, torna-se relevante aferir a validade deste instrumento para a realidade portuguesa.

Deste modo, pretende-se contribuir para o estudo da reputação social e dos comportamentos sociais em Portugal, abrindo caminho para: (a) a comparação com amostras de outros países e culturas; (b) a discriminação, com maior precisão, não apenas da valência dos comportamentos sociais, mas também de diferentes tipos entre si (por exemplo, o RCP não distinguia a retirada social da exclusão, tal como a maioria dos estudos realizados não diferencia a exclusão da vitimização; e.g., Gest, Sesma, Masten, \& Tellegen, 2006); (c) a exploração de diferentes associações entre a reputação social - e os comportamentos distintos - e o ajustamento; (d) a identificação, com maior detalhe, de possíveis situações de risco (como subgrupos de indivíduos retirados, agressivos ou vitimizados), o que permitirá não só o seu estudo para uma melhor compreensão dos fenómenos, como também o desenvolvimento de programas de intervenção mais direcionados e eficazes (Heckman, 2006).
Assim, o presente estudo tem como objetivo testar, numa amostra de pré-adolescentes e adolescentes portugueses, o último modelo proposto do $\mathrm{ECP}$ - o hexafatorial - através de uma Análise Fatorial Confirmatória, analisando igualmente se revela bom ajustamento para género e idade.

\section{Método}

\section{Participantes}

Os sujeitos que participaram neste estudo fazem parte de uma amostra de dimensão superior que se insere no âmbito de um projeto de investigação longitudinal sobre as relações sociais na adolescência. A presente amostra é constituída por 465 adolescentes (dos quais 230 são do sexo feminino e 235 do sexo masculino), com idades compreendidas entre os 11 e os $15 \operatorname{anos}(M=13,32 ; D P=1,353)$, que frequentavam o $7^{\circ}$ e $8^{\circ}$ anos em duas escolas na zona da Grande Lisboa. A taxa de consentimento dos encarregados de educação e dos próprios jovens foi superior a noventa por cento.

\section{Instrumento}

Extended Class Play. O ECP (Burgess et al., 2003) procura aceder às avaliações que os pares fazem do funcionamento e reputação social dos sujeitos. Neste questionário de 37 itens, é pedido aos adolescentes para imaginarem que são os encenadores de uma peça de teatro para a qual deverão escolher, entre os seus colegas de turma, aqueles que melhor desempenhariam diversos papéis de valência positiva e negativa. Para o efeito, é fornecida a cada sujeito uma listagem de todos os seus colegas e clarificado que, apesar de só poderem nomear um rapaz e uma rapariga para cada papel, a mesma pessoa pode ser escolhida para mais do que um.

Apenas as nomeações entre sujeitos do mesmo género são consideradas, de modo a evitar possíveis enviesamentos por estereótipos de género (Zeller et al., 2003). Os valores obtidos para os itens são estandardizados para o género e turma - para ajustar o número de nomeações recebidas ao número de nomeadores - e depois somados para cada uma das 6 dimensões avaliadas (agressividade, retirada social, comportamento pró-social, sociabilidade, vitimização e exclusão). Como já foi referido, o ECP tem demonstrado validade, fiabilidade e estabilidade em amostras normativas de jovens do $5^{\circ}$ ano em dois momentos avaliativos (por exemplo, $a$ entre 0,82 e 0,$91 ; r$ em T1 e T2 entre 0,72 e 0,83; Burgess et al., 2003; Rubin, Wojslawowicz, et al., 2006; Wojslawowicz et al., 2006).

\section{Procedimento}

Numa fase inicial, realizou-se a tradução do ECP (Burgess et al., 2003) para Português, a partir da sua versão original em Inglês. Esta adaptação obedeceu aos critérios referenciados para as traduções por Brislin (1980), designado de "abordagem por comité" (committee approach), uma metodologia para a adaptação transcultural de questionários psicológicos. Uma primeira versão foi depois 
aplicada a um pequeno grupo de pré-adolescentes, de forma a garantir que todos os itens eram compreensíveis $\mathrm{e}$, assim, adequados.

Posteriormente, foram contactadas as Direções de duas escolas da região da Grande Lisboa, tendo sido apresentados os objectivos da investigação, de modo a obter a autorização para a recolha de dados. Após a aprovação por parte das Direções, foi ainda pedida autorização aos encarregados de educação para que os seus educandos pudessem participar no estudo. Após a obtenção desta autorização e do consentimento dos alunos, teve início a recolha de dados. A administração dos instrumentos foi feita em grupo, em contexto de sala-de-aula, por dois membros da equipa de investigação treinados para o efeito. Os alunos foram informados que as suas respostas eram confidenciais. Cada sessão durou, aproximadamente, 45 minutos, tendo os adolescentes que não participaram no estudo permanecido na sala, a realizar trabalhos de casa ou outras tarefas atribuídas pelos professores.

\section{Análise Estatística}

A validade fatorial do ECP numa amostra de adolescentes portugueses foi avaliada através de uma análise fatorial confirmatória com o software AMOS (v.19, SPSS Inc, Chicago, IL), como descrito em Marôco (2010). A fiabilidade compósita e a variância extraída média (VEM) por cada fator foram avaliadas como descrito em Fornell e Larcker (1981, cit. por Marôco, 2010). A existência de outliers foi avaliada pela distância quadrada de Mahalanobis $\left(D^{2}\right)$ e a normalidade das variáveis foi avaliada pelos coeficientes de assimetria (sk) e curtose (ku) uni- e multivariada. Tendo em consideração o pressuposto da distribuição normal multivariada, foi utilizado o procedimento bootstrap, considerado um dos mais adequados quando não se verifica a regra da normalidade multivariada e quando se pretende utilizar o método da máxima verosimilhança (Efron, 1982).

A qualidade de ajustamento global do modelo fatorial foi feita de acordo com os índices e respetivos valores de referência descritos na tabela 4.1 de Marôco (2010), nomeadamente: $\chi 2 / g l$, CFI, GFI, PCFI, PGFI, RMSEA, $P[$ rmsea $\leq 0,05]$ e MECVI. A qualidade do ajustamento local foi avaliada pelos pesos fatoriais e pela fiabilidade individual dos itens, considerando-se valores superiores a 0,7 e a 0,25 como indicadores adequados, respetivamente. $\mathrm{O}$ ajustamento do modelo foi feito a partir dos índices de modificação (superiores a $11 ; p<0,001$ ) produzidos pelo AMOS e com base em considerações teóricas.

Por fim, no sentido de se testar a invariância da estrutura fatorial, pelo estudo da sua estabilidade em amostras diferenciadas, utilizou-se uma análise multigrupos (Byrne, 2000) sobre dois grupos amostrais independentes. Por outro lado, as médias dos fatores latentes entre os grupos de idade e de género foram analisados com um conjunto de modelos hierárquicos restritos comparativamente a um modelo com restrição de igualdade de médias (Marôco, 2010).

\section{Resultados}

A análise preliminar revelou que nenhuma variável apresentou valores de Sk e Ku indicadores de violações severas à distribuição Normal $(|\mathrm{Sk}|<3$ e $|\mathrm{Ku}|<10$, ver Marôco, 2010).

Seguidamente, o modelo hexafatorial do ECP original ajustado a uma amostra de 465 adolescentes revelou uma qualidade de ajustamento sofrível $\left(\chi^{2} / g l=4,57 ; \mathrm{CFI}=0,83\right.$; $\mathrm{GFI}=0,80 ;$ PCFI $=0,74 ;$ PGFI $=0,66 ;$ RMSEA $=0,09$; $P[r m s e a \leq 0,05]<0,001 ; \mathrm{MECVI}=3,13)$. Este modelo foi comparado, tal como sugere Thompson (2004, cit. por Marôco, 2010), com modelos rivais, concretamente com o de fatores não correlacionados, tendo este revelado um mau ajustamento - o que permitiu excluí-lo enquanto modelo plausível $\left(\chi^{2} / g l=6,41 ; \mathrm{CFI}=0,74 ; \mathrm{GFI}=0,73 ; \mathrm{PCFI}=0,78\right.$; PGFI $=0,63$; RMSEA $=0,11 ; P[$ rmsea $\leq 0,05]<0,001)$. De modo a melhorar o ajustamento do modelo, foram eliminadas quarenta e cinco observações outliers( $p 1$ e $p 2<0,001)$, obtendo-se uma qualidade de ajustamento algo sofrível $\left(\chi^{2}\right)$ $g l=3,46 ; \mathrm{CFI}=0,85 ; \mathrm{GFI}=0,83 ; \mathrm{PCFI}=0,75 ; \mathrm{PGFI}=0,68$; RMSEA $=0,08 ; P[$ rmsea $\leq 0,05]<0,001)$.

De seguida, procedeu-se à remoção de alguns itens que, do ponto de vista teórico, não pareciam representar e distinguir claramente o comportamento social a ser estudado e cujos índices de modificação (IM) não só apresentavam valores superiores a 11 (aumentando assim a probabilidade de erro tipo I), como sugeriam a saturação em fatores diferentes dos sugeridos na versão original do ECP. Assim, de modo averiguar se o ajustamento do modelo poderia ser melhorado, foram eliminados os itens 12 ("sabe esperar pela sua vez") e 37 ("é bem-educado") do fator "Comportamento pró-social", o 17 ("é considerado, respeitado e cujas opiniões são ouvidas por todos") da "Sociabilidade/ Popularidade", o 23 ("é muito mandão") da "Agressividade" e, ainda, o 34 ("fica nervoso quando participa em discussões de grupo") relativo ao da Retirada Social.

Adicionalmente, foram também incluídas trajetórias no modelo entre os resíduos dos itens 10 e 25 (fator "Comportamento pró-social"), 6 e 22 ("Sociabilidade/ Popularidade"), bem como 7 e 11 e 29 e 36 ("Agressividade"). Desta forma, obteve-se uma qualidade de ajustamento bastante boa $\left(\chi^{2} / g l=1,95 ; \mathrm{CFI}=0,95 ; \mathrm{GFI}=0,92 ; \mathrm{PCFI}=0,80\right.$; PGFI $=0,71$; RMSEA $=0,05 ; P[$ rmsea $\leq 0,05]<0,71$; ME$\mathrm{CVI}=1,31)$, em suporte da validade hexafatorial do ECP, passando este modelo, em função dos bons valores de ajustamento registados, a ser assumido como o Modelo Final (MF). Um teste de diferenças de $\chi^{2}$ permitiu concluir que a qualidade de ajustamento deste modelo final é significativamente superior à do modelo original na amostra sob estudo $\left(\chi^{2} \operatorname{dif}(124)=746,56, p<0,05\right)$, apresentando igualmente um MECVI consideravelmente menor (1,31 vs. 3,13), ou seja, é não só de melhor qualidade, como também mais parcimonioso.

Demonstrada a adequação da estrutura fatorial proposta para a amostra em estudo, também a fiabilidade compósita 
Correia, J. V., Santos, A. J., Freitas, M., Rosado, A. \& Rubin, K. (2014). Análise Fatorial Confirmatória do Extended Class Play numa Amostra Portuguesa de Jovens Adolescentes.

dos fatores, que estima a consistência interna dos itens reflexivos dos construtos ou fatores, se revelou elevada (Tabela 1). Com efeito, todos os valores registados se situam acima de 0,7 - valor indicador de fiabilidade apropriada-, sendo de 0,90 para a "Agressividade", 0,80 para "Retirada social", 0,79 para "Comportamento pró-social", 0,77 para "Sociabilidade/Popularidade", 0,81 para "Exclusão" e, finalmente, 0,93 para "Vitimização".

A variância extraída média (VEM) - um indicador de validade convergente dos fatores - revelou-se igualmente adequada (Tabela 1), apresentando todos os fatores valores iguais ou superiores a 0,5 (0,59 para "Agressividade"; 0,57 para "Retirada social"; 0,49 para "Comportamento pró-social"; 0,46 para "Sociabilidade/ Popularidade"; 0,59 para "Exclusão"; e 0,81 para "Vitimização"). Também a validade discriminante de todos os fatores foi demonstrada, na medida em que as suas correlações ao quadrado são consideravelmente inferiores aos valores de VEM de cada um dos mesmos (Tabela 1). Desta forma, conclui-se que os itens que reflectem um fator não estão correlacionados com outros fatores - ou, de outro modo, que os fatores, definidos por cada conjunto de itens, são distintos entre si.

Tabela 1

Fiabilidade Compósita, Validade Convergente e Discriminante das Dimensões do ECP

\begin{tabular}{|c|c|c|c|c|c|c|c|c|c|}
\hline & & \multirow{2}{*}{$\mathrm{FC}$} & \multirow{2}{*}{ VEM } & \multicolumn{6}{|c|}{$r^{2}$} \\
\hline & & & & 1. & 2. & 3. & 4. & 5. & 6. \\
\hline & Agressividade & 0,90 & 0,59 & & & & & & \\
\hline & Retirada Social & 0,80 & 0,57 & 0,03 & & & & & \\
\hline & Comp. Pró-social & 0,79 & 0,81 & 0,01 & 0,00 & & & & \\
\hline & Sociabilidade & 0,77 & 0,46 & 0,03 & 0,03 & 0,38 & & & \\
\hline & Exclusão & 0,81 & 0,59 & 0,01 & 0,39 & 0,02 & 0,07 & & \\
\hline & Vitimização & 0,93 & 0,81 & 0,05 & 0,04 & 0,01 & 0,03 & 0,58 & \\
\hline
\end{tabular}

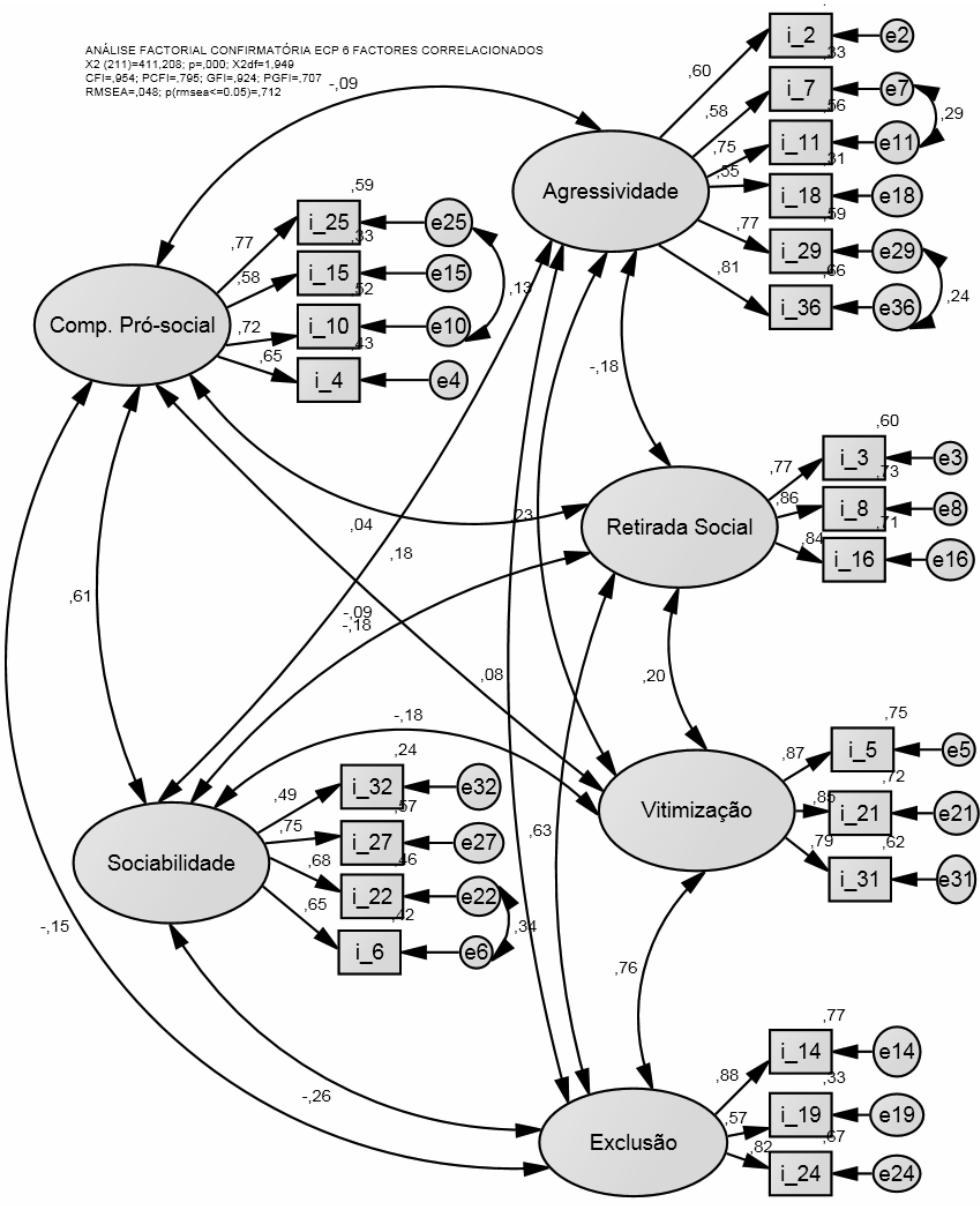

Figura 1. Modelo final do ECP ajustado a uma amostra de 465 adolescentes portugueses. Nota. $\left(X^{2} / g l=1,949 ; \mathrm{CFI}=0,954 ;\right.$ PCFI $=0,795$; $\mathrm{GFI}=0,924 ; \mathrm{PGFI}=0,707$; RMSEA $=0,048$; $P[$ rmsea $\leq 0,05]<0,712 ; \mathrm{AIC}=541,208 ; \mathrm{ME}-$ $\mathrm{CVI}=1,311)$. 
A Figura 1 apresenta os valores dos pesos fatoriais estandardizados e a fiabilidade individual de cada um dos itens no modelo final simplificado. Como se pode constatar, todos os itens apresentam pesos fatoriais estandardizados iguais ou superiores a 0,5 , revelando que todos os fatores apresentam validade fatorial. A observação da Figura 1 e da Tabela 2 permite ainda analisar a associação entre os fatores do ECP através das suas correlações. Com efeito, verifica-se um padrão de associação entre eles, do qual salientamos a correlação entre as dimensões da Sociabilidade e do Comportamento Pró-social $(r=0,61)$, Agressividade e Vitimização $(r=0,23)$, Exclusão com Retirada Social $(r=0,63)$ e Sociabilidade $(r=-0,26)$. Finalmente, destacamos a associação entre as dimensões da Exclusão e da Vitimização $(r=0,77)$, indicadora de que estes dois construtos, embora se correlacionem fortemente, são independentes - o que reforça a precisão do modelo hexafatorial em estudo.

Tabela 2

Correlações entre as Dimensões do ECP

$\begin{array}{llllll}1 . & 2 . & 3 . & 4 . & 5 . & 6 .\end{array}$

\begin{tabular}{|c|c|c|c|c|c|c|}
\hline 1. & Agressividade & & & & & \\
\hline 2. & Retirada Social & $-0,18^{*}$ & & & & \\
\hline 3. & Comp. Pró-social & $-0,09$ & 0,04 & & & \\
\hline 4. & Sociabilidade & $0,18^{*}$ & $-0,18^{*}$ & $0,61 * *$ & & \\
\hline 5. & Exclusão & 0,08 & $0,63 * * *$ & $-0,15^{*}$ & $-0,26^{* *}$ & \\
\hline 6. & Vitimização & $0,23 * *$ & $0,20 * *$ & $-0,09$ & $-0,18^{* *}$ & $0,77 * * *$ \\
\hline
\end{tabular}

$* \mathrm{p}<0.05 ; * * \mathrm{p}<0.01 ; * * * \mathrm{p}<0.001$

\section{Comparação em Função do Género e Idade}

Finalmente e de modo a avaliar possíveis variações nesta estrutura fatorial consoante o género e a idade foram efetuadas duas análises multigrupos - para raparigas e rapazes, bem como para sujeitos mais novos (idade inferior a 13 anos) e mais velhos (idade superior ou igual a 13 anos). A invariância do modelo foi, assim, avaliada em ambas as análises por comparação do modelo livre (com pesos fatoriais e variâncias/covariâncias dos fatores livres) com um modelo constrito onde foram fixados os pesos fatoriais e as variâncias/covariâncias dos grupos. A significância estatística da diferença dos modelos foi feita com o teste do Qui-quadrado, como descrito em Marôco (2010), sendo os resultados apresentados na Tabela 3.

A análise multigrupos conduzida para o sexo revelou que o modelo constrito com pesos fatoriais fixos nas raparigas $v s$. rapazes apresentou um ajustamento significativamente pior do que o modelo com parâmetros livres $\left(\chi^{2} \operatorname{dif}(17)=29,495, p=0,030\right)$. No entanto, o modelo com coeficientes estruturais fixos não diferiu significativamente do modelo com coeficientes estruturais livres, revelando, desse modo, invariância $\left(\chi^{2} \operatorname{dif}(21)=31,78, p=0,062\right)$. Adicionalmente, também a análise das diferenças significativas entre as médias dos dois grupos não se revelou significativa $\left(\chi^{2} \operatorname{dif}(40)=43,95, p=0,308\right)$. Assim, os resultados evidenciam a variância do modelo nos pesos fatoriais de apenas alguns itens sobre os respectivos fatores. Um teste $Z$ à igualdade dos coeficientes estruturais revelou que os coeficientes referentes às trajetórias $\mathrm{i} 2<$-Agressividade $(Z=2,04 ; p<0,05)$, i16<-Retirada Social $(Z=-4,17 ; p<0,05)$, i5<-Vitimização $(Z=-3,44 ; p<0,05)$, i2 $1<$-Vitimização $(Z=-3,83 ; p<0,05)$, i35<-Vitimização $(Z=-2,12 ; p<0,05)$ e i14<-Exclusão $(Z=-2,08 ; p<0,05)$ diferiram significativamente entre os dois grupos. Em conclusão, podemos afirmar que, embora os pesos fatoriais de alguns itens apresentem variância entre os grupos, a estrutura fatorial do modelo final é estável entre raparigas e rapazes.

$\mathrm{Na}$ análise multigrupos conduzida para a idade, o teste de Qui-Quadrado $\left(\chi^{2}\right)$ apresentou diferenças significativas apenas entre o modelo livre e o modelo com pesos fatoriais fixos $\left(\chi^{2} \operatorname{dif}(23)=91,98, p<0,001\right)$. Pelo contrário, a comparação do modelo livre com os modelos em que as variâncias/covariâncias e as médias foram fixas revelou invariância $\left(\chi^{2} \operatorname{dif}(15)=14,56, p=0,483 ; X^{2} \operatorname{dif}(23)=33,37, p=0,075\right.$, respetivamente). Desta forma, mais uma vez, os resultados demonstram a variância do modelo meramente nos pesos fatoriais de alguns itens sobre os respectivos fatores. Um teste $Z$ à igualdade dos coeficientes estruturais revelou que os coeficientes referentes às trajetórias i $4<$ - Pró-social $(Z=-2,94 ; p<0,05)$, i6<-Sociabilidade $(Z=3,24 ; p<0,05)$, i22 $<$ - Sociabilidade $(Z=2,45 ; p<0,05)$, i36 $<$-Agressividade $(Z=2,17 ; p<0,05)$, i3<-Retirada Social $(Z=2,55 ; p<0,05)$, i8 $<$-Retirada Social $(Z=2,88, p<0,05)$, i21<-Vitimização $(Z=3,16 ; p<0,05)$, i14<-Exclusão $(Z=4,91 ; p<0,05)$, i24<Exclusão $(Z=3,64 ; p<0,05)$ diferiram significativamente entre os dois grupos. 
Correia, J. V., Santos, A. J., Freitas, M., Rosado, A. \& Rubin, K. (2014). Análise Fatorial Confirmatória do Extended Class Play numa Amostra Portuguesa de Jovens Adolescentes.

Tabela 3

Resultados da Análise Multigrupos por Sexo e Idade

\begin{tabular}{lcccccc}
\hline & \multicolumn{3}{c}{ Sexo } & \multicolumn{3}{c}{ Idade } \\
\hline Modelo Final & $G L$ & CMIN & $p$ & $G L$ & CMIN & $p$ \\
Pesos fatoriais & 17 & 29,495 & 0,030 & 23 & 91,981 & 0,000 \\
Covariâncias Estruturais & 21 & 31,784 & 0,062 & 15 & 14,562 & 0,483 \\
Médias & 40 & 43,949 & 0,308 & 23 & 33,367 & 0,075 \\
\hline
\end{tabular}

\section{Discussão}

Os nossos resultados suportam a estrutura hexafatorial original da escala, embora com algumas diferenças no número de itens. De facto, os procedimentos - realizados com base em critérios estatísticos e teóricos - como a eliminação de observações outliers e de itens com pesos fatoriais inaceitáveis, ou a correlação entre erros de medida de itens pertencentes a um mesmo fator permitiram a emergência de um modelo hexafatorial que revelou uma boa qualidade de ajustamento global e local. Demonstrou também bons níveis de fiabilidade compósita e validade fatorial, convergente e discriminante, assim como maior parcimónia do que o modelo original investigado, pelo que foi adotado como o modelo final.

Esta estrutura apresenta não só valores indicadores de qualidade de ajustamento superiores aos apresentados por Burgess et al. (2003; Burgess et al., 2004) para a estrutura de cinco fatores, como ainda permite uma melhor distinção entre constructos conceptualmente diferentes. De facto, os nossos resultados dão suporte a esta estrutura hexafatorial, onde não só existe maior diferenciação entre tipos distintos de agressividade e isolamento social, como também se distinguemas dimensões da exclusão social e da vitimização, à semelhança do que tem sido sugerido na investigação mais recente. Com efeito, está demonstrado que a rejeição pelos pares é um preditor significativo do evitamento da escola, da diminuição da participação nas atividades escolares, bem como de dificuldades de internalização (e.g. Buhs et al., 2010; Gazelle \& Rudolph, 2004). No entanto, os trabalhos que procuram adotar um olhar mais preciso deste fenómeno têm indicado padrões de associação diferentes para as duas formas que a rejeição pelos pares pode assumir: em particular, a agressividade tem sido sugerida como associada à vitimização, enquanto a retirada social tem sido associada, por seu turno, à exclusão social (Gazelle \& Ladd, 2003; Menzer et al., 2010).

$\mathrm{O}$ recente modelo do ECP permite, assim, um estudo mais minucioso do funcionamento daqueles sujeitos que apresentam dificuldades nas experiências com os pares, seja por se retirarem eles próprios da interação com o grupo de pares, de forma recorrente e consistente ao longo de diferentes situações, contextos e tempo (Coplan, \& Rubin, 2008; Rubin, 1982; Rubin et al., 2009) - os jovens socialmente retirados - ou por serem mantidos à margem do grupo porque os pares rejeitam as suas tentativas de interação - os jovens socialmente excluídos (Rubin, Bukowski, et al., 2006; Rubin \& Mills, 1988).

Adicionalmente, a estrutura hexafatorial sob estudo revelou ainda boa qualidade de ajustamento para género e idade. De facto, quando o modelo foi testado separadamente para raparigas e rapazes, bem como para sujeitos mais novos e mais velhos, a estrutura global manteve-se estável e adequada aos dados, revelando que a estrutura é equivalente nestes grupos de género e de idade. De particular importância é a evidência de que os coeficientes ou trajetórias estruturais entre os fatores latentes é invariante em função dos grupos em análise e que, adicionalmente, as médias das variáveis latentes também são equivalentes. Os itens reflexos de cada fator mantêm-se idênticos, embora com alguma variação nos pesos fatoriais de alguns índices. Assim, os nossos resultados parecem demarcar-se de outros trabalhos que dão conta de diferenças de género ou de idade no comportamento social dos sujeitos, como, por exemplo, maiores níveis de agressividade nos rapazes (e.g. Gazelle \& Rudolph, 2004; Rose \& Smith, 2009; Rubin et al., 2009; Rubin, Wojslawowicz, et al., 2006). No entanto, é importante salientar que esses dados resultam maioritariamente de amostras mais novas, pelo que será necessário o aprofundamento de estudos na adolescência. Deste modo, este instrumento afigura-se como um importante método de avaliação do funcionamento, comportamento ou reputação social dos jovens portugueses, revelando várias vantagens.

A primeira prende-se com o facto de as nomeações de pares serem um procedimento que permite captar as perspetivas daqueles que determinam, de facto, o estatuto e reputação social de cada indivíduo (Hymel \& Rubin, 1985), de uma forma que os adolescentes habitualmente gostam, na medida em que não é muito diferente das avaliações que fazem diariamente e pelas quais regulam o seu comportamento. Também todas as transformações cognitivas e sociais normativas da adolescência colocam os pares numa posição privilegiada de melhores informadores sobre os comportamentos dos colegas. Efetivamente, se as primeiras permitem uma maior acuidade e validade dos seus relatos, um dos traços distintivos desta fase é o facto de a maior parte das interações passar a ocorrer fora do 
alcance dos adultos, na privacidade, intimidade e multiplicidade de contextos a que apenas os pares têm acesso. Tal constitui-se como uma enorme vantagem deste método, comparativamente a outros que confiam na observação ou em relatos de pais e/ ou professores: a possibilidade de obter, de forma mais eficaz e provavelmente mais válida, a perspetiva que os diversos colegas têm sobre uma grande variedade de comportamentos sociais que os adolescentes assumem, de forma espontânea, em contextos fora do espaço escolar e familiar.

Neste sentido, o ECP oferece uma visão multidimensional do funcionamento social, o que possibilita uma melhor caracterização e um estudo mais completo do comportamento e experiências sociais dos sujeitos (em particular, na adolescência) do que medidas como, por exemplo, a popularidade ou aceitação social. Por outro lado, este método permite a recolha de informações sobre muitos sujeitos de forma relativamente rápida. Tal poderá ser importante no estudo da reputação (e das suas diferentes dimensões) e do ajustamento psicossocial dos alunos no geral e, até, de fenómenos pouco investigados na realidade portuguesa, como o da retirada social. Efetivamente, é importante, em estudos futuros, identificar, com base nas perceções dos colegas, aqueles que se mantêm à margem do grupo devido a timidez e ansiedade social e, assim, poder não só caracterizá-los a nível individual, relacional e grupal, como também avaliar as trajetórias e consequências desenvolvimentais desta menor interação com os pares. Estes trabalhos, que têm sido realizados essencialmente na América do Norte (e.g. Booth-LaForce et al., 2012; Menzer et al., 2010; Oh et al., 2008; Rubin, Bukowski, et al., 2006), poderão agora ser levados a cabo em países de expressão portuguesa.

Este método permitirá, ainda, pela facilidade da sua aplicação, estudar muitos adolescentes, de diferentes escolas, idades, etnias e contextos socioeconómicos, bem como comparar os dados obtidos na realidade portuguesa com os de outras culturas, aferindo pontos comuns e específicos. Desta forma, o ECP poderá ser um importante auxiliar na deteção e identificação de dificuldades dos jovens no domínio social e dos problemas de ajustamento que lhes estão associados e, assim, poderá contribuir para o desenvolvimento de programas de prevenção (e.g., bullying, agressividade, retirada social) ou de promoção de competências.

Os resultados aqui apresentados sustentam a estrutura hexafatorial do ECP, ainda que julguemos ser importante a avaliação da estabilidade do modelo ao longo do tempo e a sua replicação - desejavelmente com uma amostra não só de dimensão superior, mas também com características socioeconómicas e geográficas mais heterogéneas. Poderá ser igualmente interessante averiguar a concordância com outras medidas, nomeadamente as de avaliação de pais e professores sobre o comportamento e funcionamento social. Assim, é também aconselhado o desenvolvimento do estudo da validade concorrente ou da validade preditiva do ECP.

\section{Referências}

Bagwell, C. L., Newcomb, A. F., \& Bukowski, W. M. (1998). Preadolescent friendship and peer rejection as predictors of adult adjustment. Child Development, 69, 140-153.

Booth-LaForce, C., Oh, W., Kennedy, A. E., Rubin, K. H., RoseKrasnor, L., \& Laursen, B. (2012). Parent and peer links to trajectories of anxious withdrawal from grades 5-8. Journal of Clinical Child and Adolescent Psychology, 41, 138-149.

Brislin, R. (1980). Translation and content analysis for oral and written material. In H. Triandis \& J. Berry (Eds.), Handbook of cross-cultural psychology (Vol. 2, pp. 389-444). Needham Heights, MA: Allyn and Bacon.

Buhs, E., Ladd, G., \& Herald-Brown, S. (2010). Victimization and exclusion: Links to peer rejection, classroom engagement, and achievement. In S. R. Jimerson \& S. M. Swearer (Eds.), Handbook of bullying in schools: An international perspective (pp. 163-172). New York: Routledge.

Burgess, K. B., Rubin, K. H., Wojslawowicz, J. C., Rose-Krasnor, L., \& Booth, C. L. (2003). The "Extended Class Play": A longitudinal study of its factor structure, reliability, and validity. Paper presented at the biennial meeting of the Society for Research in Child Development, Tampa, FL, USA.

Burgess, K. B., Wojslawowicz Bowker, J. C., Oh, W., Rubin, K. H., Booth-LaForce, C., \& Rose-Krasnor, L. (2004). The "Extended Class Play": A longitudinal study of its factor structure, reliability, and validity. Unpublished manuscript under review.

Burt, K. B., Obradović, J., Long, J. D., \& Masten, A. S. (2008). The interplay of social competence and psychopathology over 20 years: Testing transactional and cascade models. Child Development, 79(2), 359-374.

Byrne, B. M. (2000). Structural equation modelling with AMOS: Basic concepts, applications, and programming. Mahwah, NJ: Lawrence Erlbaum.

Casiglia, A. C., Lo Coco, A., \& Zappulla, C. (1998). Aspects of social reputation and peer relationships in Italian children: A cross-cultural perspective. Developmental Psychology, 34, 723-730.

Chen, X., Chung, J., \& Hsiao, C. (2009). Peer interactions and relationships from a cross-cultural perspective. In K. H. Rubin, W. M. Bukowski, \& B. Laursen (Eds.), Handbook of peer interactions, relationships, and groups (pp. 379-393). New York: Guilford Press.

Chen, X., Rubin, K. H., Li, B., \& Li, D. (1999). Adolescent outcomes of social functioning in Chinese children. International Journal of Behavioral Development, 23(1), 199-223.

Chen, X., Rubin, K. H., \& Sun, Y. (1992). Social reputation and peer relationships in Chinese and Canadian children. Social Development, 3, 269-290.

Coplan, R. J., \& Rubin, K. H. (2008). Social withdrawal in childhood: Conceptual approaches, definitions, and methodological issues. In A. LoCoco, K. H. Rubin, \& C. Zappulla (Eds.), L'isolamento sociale durante l'infanzia. Milano, Italy: Unicopli.

Efron, B. (1982). The Jackknife, the bootstrap and other resampling plans. Philadelphia, PA: Society for Industrial and Applied Mathematics.

Gazelle, H., \& Ladd, G. W. (2003). Anxious solitude and peer exclusion: A diathesis-stress model of internalizing trajectories in childhood. Child Development, 74, 257-278. 
Correia, J. V., Santos, A. J., Freitas, M., Rosado, A. \& Rubin, K. (2014). Análise Fatorial Confirmatória do Extended Class Play numa Amostra Portuguesa de Jovens Adolescentes.

Gazelle, H., \& Rudolph, K. (2004). Moving toward and away from the world: Social approach and avoidance trajectories in anxious solitary youth. Child Development, 75, 829-849.

Gest, S., Sesma, A., Masten, A., \& Tellegen, A. (2006). Childhood peer reputation as a predictor of competence and symptoms 10 years later. Journal of Abnormal Child Psychology, 34, 509-526.

Gifford-Smith, M. E., \& Brownell, C. A. (2003). Childhood peer relationships: Social acceptance, friendships, and peer networks. Journal of School Psychology, 41, 235-284.

Harter, S. (1993). Causes and consequences of low self-esteem in children and adolescents. In R. F. Baumeister (Ed.), Selfesteem: The puzzle of low self-regard (pp. 87-116). New York: Plenum Press.

Hartup, W. W. (1992). Social relationships and their developmental significance. American Psychologist, 44, 120-126.

Heckman, J. J. (2006). Skill formation and the economics of investing in disadvantaged children. Science, 312, 1900-1902.

Hymel, S., \& Rubin, K. H. (1985). Children with peer relationship and social skills problems: Conceptual, methodological, and developmental issues. In G. J. Whitehurst (Ed.), Annals of child development (Vol. 2, pp. 251-297). Greenwich, CT: JAI Press.

Hymel, S., Wagner, E., \& Butler, L. (1990). Reputational bias: View from the peer group. In S. R. Asher \& J. Coie (Eds.), Peer rejection in childhood (pp. 156-186). Cambridge, UK: Cambridge University Press.

Luthar, S. S., \& McMahon, T. J. (1996). Peer reputation among inner-city adolescents: Structure and correlates. Journal of Research on Adolescence, 6(4), 581-603.

Marôco, J. (2010). Análise de equações estruturais: Fundamentos teóricos, software e aplicações. Pêro Pinheiro, Portugal: ReportNumber.

Masten, A. S. (2005). Peer relationships and psychopathology in developmental perspective: Reflections on progress and promise. Journal of Clinical Child \& Adolescent Psychology, 34(1), 87-92.

Masten, A. S., \& Coatsworth, J. D. (1998). The development of competence in favorable and unfavorable environments: Lessons from research on successful children. American Psychologist, 53(2), 205-220.

Masten, A. S., \& Curtis, W. J. (2000). Integrating competence and psychopathology: Pathways toward a comprehensive science of adaptation in development. Development and Psychopathology, 12, 529-550.

Masten, A., Morison, P., \& Pellegrini, D. (1985). A revised Class Play Method of Peer Assessment. Developmental Psychology, 21(3), 523-533.

Menzer, M. M., Oh, W., McDonald, K. L., Rubin, K. H., \& Dashiell-Aje, E. (2010). Behavioral correlates of peer exclusion and victmization of East Asian American and European American young adolescents. Asian American Journal of Psychology, 4, 290-302.

Morison, P., \& Masten, A. S. (1991). Peer reputation in middle childhood as a predictor of adaptation in adolescence; A seven-year follow-up. Child Development, 62, 991-1007.

Moskowitz, D. S., Schwartzman, A. E., \& Ledingham, J. E. (1985). Stability and change in aggression and withdrawal in middle childhood and early adolescence. Journal of Abnormal Psychology, 94(1), 30-41.

Oh, W., Rubin, K., Bowker, J., Booth-LaForce, C., Rose-Krasnor, L., \& Laursen, B. (2008). Trajectories of social withdrawal middle childhood to early adolescence. Journal of Abnormal Child Psychology, 36, 553-566.
Prinstein, M. J., Racourt, D., Guerry, J. D., \& Browne, C. B. (2009). Peer reputations and psychological adjustment. In K. H. Rubin, W. Bukowski, \& B. Laursen (Eds.), The handbook of peer interactions, relationships, and groups (pp. 82-99). New York: Guilford.

Roeser, R. W., Eccles, J. S., \& Sameroff, A. J. (2000). School as a context of early adolescents' academic and social-emotional development: A summary of research findings. Elementary School Journal, 100(5), 443-471.

Rose, A., \& Smith, R. (2009). Sex differences in peer relationships. In K. H. Rubin, W. M. Bukowski, \& B. Laursen (Eds.), Handbook of peer interactions, relationships, and groups (pp. 379-393). New York: Guilford Press.

Rubin, K. H. (1982). Non-social play in preschoolers: Necessary evil? Child Development, 53, 651-657.

Rubin, K. H., Bukowski, W., \& Parker, J. G. (2006). Peer interactions, relationships, and groups. In W. Damon, R. M. Lerner \& N. Eisenberg (Eds.), Handbook child psychology: Social, emotional, and personality development ( $6^{\text {th }}$ ed., pp. 571-645) New York: Wiley.

Rubin, K., Cheah, C., \& Menzer, M. (2010). Peers. In M. H. Bornstein (Ed.), Handbook of cultural developmental science (pp. 223-237). New York: Psychology Press.

Rubin, K. H., Chen, X., McDougall, P., Bowker, A., \& McKinnon, J. (1995). The Waterloo Longitudinal Project: Predicting internalizing and externalizing problems in adolescence. Development and Psychopathology, 7, 751-764.

Rubin, K. H., Coplan, R. J., \& Bowker, J. C. (2009). Social withdrawal in childhood. Annual Review of Psychology, 60(1), 141-171.

Rubin, K. H., Coplan, R. J., Chen, X., Buskirk, A. A., \& Wojslawowicz, J. D. (2005). Peer relationships in childhood. In M. H. Bornstein \& M. E. Lamb (Eds.), Developmental psychology: An advanced textbook ( $5^{\text {th }}$ ed., pp. 469-512). Mahwah, NJ Lawrence Erlbaum.

Rubin, K. H., \& Mills, R. (1988). The many faces of social isolation in childhood. Journal of Consulting and Clinical Psychology, 6, 916-924.

Rubin, K. H., Wojslawowicz, J. C., Burgess, K. B., Rose-Krasnor, L., \& Booth-LaForce, C. L. (2006). The best friendships of shy/withdrawn children: Prevalence, stability, and relationship quality. Journal of Abnormal Child Psychology, 34, 139-153.

Sullivan, H. S. (1953). The interpersonal theory of psychiatry. New York: Norton.

Trentini, G., \& Muzio, G. B. (1995). Values in a cross-cultural perspective: A further analysis. In V. Sverko \& B. Super (Eds.), Life roles, values, and careers (pp. 241-251). San Francisco, CA: Jossey-Bass.

Wojslawowicz, J., Rubin, K., Burgess, K., Booth-Laforce, C., \& Rose-Krasnor, L. (2006). Behavioral characteristics associated with stable and fluid best friendship patterns in middle childhood. Merrill-Palmer Quarterly: Journal of Developmental Psychology, 52, 671-693.

Zeller, M., Vannatta, K., Schafer, J., \& Noll, R. B. (2003). Behavioral reputation: A cross-age perspective. Developmental Psychology, 39(1), 129-139. 